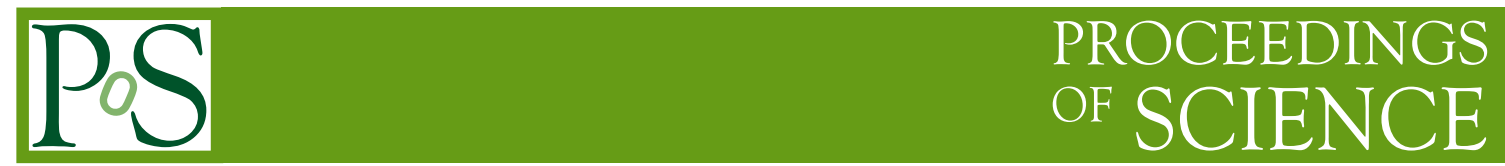

\title{
Soft gluon rescattering in diffractive DIS
}

\section{Roman Pasechnik*, Rikard Enberg, and Gunnar Ingelman}

Department of Physics and Astronomy, Uppsala University, Box 516, SE-751 20 Uppsala, Sweden

We construct a QCD-based model where soft gluon rescattering between final state partons in deep inelastic scattering leads to events with large rapidity gaps and a leading proton. The model successfully describes the precise HERA data on the diffractive deep inelastic structure function in the whole available kinematical range.

XVIII International Workshop on Deep-Inelastic Scattering and Related Subjects, DIS 2010 April 19-23, 2010

Firenze, Italy

\footnotetext{
${ }^{*}$ Speaker.
} 


\section{Introduction}

Diffractive processes are sensitive to the details of nonperturbative QCD dynamics and provide a way to probe the soft and semihard regimes directly. Diffractive events are characterized by a leading "target" particle, carrying most of the beam momentum, and a well separated produced hadronic system. The "gap" in between is connected to the soft part of the event and therefore to nonperturbative effects at a long space-time scale. Diffractive deep inelastic scattering (DDIS) offers a particularly good opportunity to explore the interplay between hard and soft physics due to the precise data from the electron-proton collider HERA [ [ [ ㅁ].

A dynamical interpretation of hard diffraction was initially proposed in Ref. [B]]. This Soft Color Interaction (SCI) model is based on the simple assumption of soft gluon exchanges leading to color rearrangements between the final state partons. Variations in the topology of the confining color fields lead to different hadronic final states, including those with rapidity gaps.

The only parameter of this model is the probability for a soft exchange, accounting for the unknown nonperturbative dynamics. Remarkably, the SCI model is phenomenologically very successful in describing many different processes, both diffractive and nondiffractive [䧃], with only a single parameter $P \simeq 0.5$ for this probability. Thus, the SCI model captures the essential dynamics of diffraction, but lacks a solid theoretical basis.

To understand better what we can learn from the phenomenology of the SCI model, we discuss here a QCD-based mechanism for soft gluon rescattering of final state partons. This mechanism leads to effective color singlet exchange and thereby to diffractive scattering. Our model was initially introduced in a recent letter [[]], and was presented in Ref. [6] in more detail. Here we give the essence of this new model and discuss its potential.

\section{Diffractive structure function}

Let us consider first the simplest case of the $q \bar{q}$ contribution shown in Fig. 1 (left), which is the leading one for small invariant mass of $X$ system $M_{X}$.
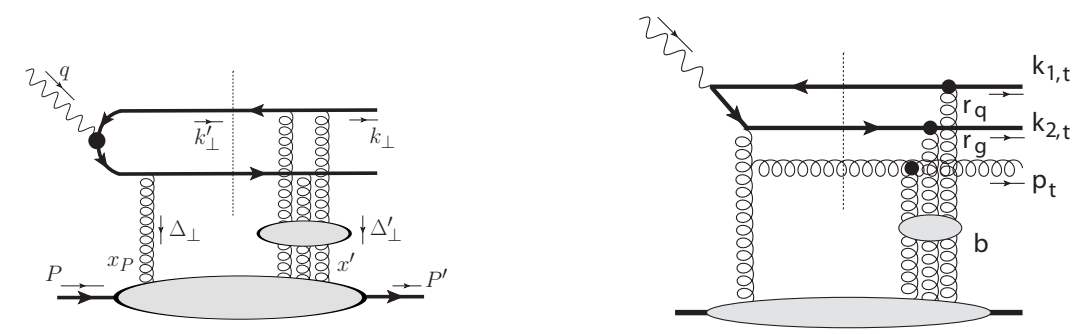

Figure 1: Amplitude of the process $\gamma^{*} p \rightarrow X p$ with $q \bar{q}$ dipole scattering contribution with all-order resummed soft gluon exchange (left) and gluon emission (right) giving a non-negligible contribution for large $M_{X}($ small $\beta)$.

As was demonstrated in Refs. [1], 团, the total amplitude of $\gamma^{*} P \rightarrow X P^{\prime}$ process can be decomposed in the configuration space into the hard part, which describes the coupling of the hard gluon carrying the most of the momentum transfer $x_{P} P$ between the $q \bar{q}$ dipole and the proton target, and 
the soft part containing information about (almost) real quark/antiquark rescattering off the proton remnants, i.e.

$$
M(\boldsymbol{\delta}) \sim \int d^{2} b e^{-i \boldsymbol{\delta} \mathbf{b}} \hat{M}^{\text {hard }}(\mathbf{b}) \hat{M}^{\text {soft }}(\mathbf{b})
$$

QCD factorization of the process is provided by the hard scale $\mu_{F}^{2}$ being the hardest quark virtuality $\mu_{F}^{2} \equiv \varepsilon^{2}+k_{\perp}^{2}=z(1-z)\left(M_{X}^{2}+Q^{2}\right)$, where $\varepsilon=\sqrt{z(1-z)} Q$ and $k_{\perp}=\sqrt{z(1-z)} M_{X}$ are the energy and transverse momentum of a quark/antiquark.

The Fourier-transformed amplitudes $\hat{M}^{\text {hard }}$ and $\hat{M}^{\text {soft }}$ were derived in Ref. [焑]. Here we only list the final results for the longitudinal and transverse contributions to the fully-unintegrated diffractive structure functions $F_{L, T}^{D,(4)}\left(x_{P}, Q^{2}, \beta, t\right)$ :

$$
\begin{aligned}
& x_{P} F_{L}^{D(4)}=\mathscr{S} Q^{4} M_{X}^{2} \int_{z_{\text {min }}}^{\frac{1}{2}} d z(1-2 z) z^{2}(1-z)^{2}\left|J_{L}\right|^{2} \\
& x_{P} F_{T}^{D(4)}=2 \mathscr{S} Q^{4} \int_{z_{\text {min }}}^{\frac{1}{2}} d z(1-2 z)\left\{(1-z)^{2}+z^{2}\right\}\left|J_{T}\right|^{2},
\end{aligned}
$$

where $\mathscr{S}=\sum_{q} e_{q}^{2} /\left(2 \pi^{2} N_{c}^{3}\right)$ sums over light quark charges $e_{q}$, and

$$
\begin{aligned}
& J_{L}=i \alpha_{s}\left(\mu_{F}^{2}\right) \int d^{2} \mathbf{r} d^{2} \mathbf{b} e^{-i \boldsymbol{\delta} \mathbf{b}} e^{-i \mathbf{r} \mathbf{k}_{\perp}} K_{0}(\varepsilon r) \mathscr{V}(\mathbf{b}, \mathbf{r})\left[1-e^{\mathscr{A} \mathscr{W}}\right] \\
& J_{T}=i \alpha_{s}\left(\mu_{F}^{2}\right) \int d^{2} \mathbf{r} d^{2} \mathbf{b} e^{-i \boldsymbol{\delta} \mathbf{b}} e^{-i \mathbf{r k}_{\perp}} \varepsilon K_{1}(\varepsilon r) \frac{r_{x} \pm i r_{y}}{r} \mathscr{V}(\mathbf{b}, \mathbf{r})\left[1-e^{\mathscr{A} \mathscr{W}}\right] .
\end{aligned}
$$

where expression in square brackets represents the result of exponentiation of soft gluon final-state interactions in the large $N_{c}$ limit, with $\mathscr{A}=2 \pi i C_{F} \alpha_{s}\left(\mu_{\text {soft }}^{2}\right)$ and $\mathscr{W}=(1 / 2 \pi) \ln (|\mathbf{b}-\mathbf{r}| /|\mathbf{b}|), K_{0,1}$ are the Bessel functions, and

$$
\mathscr{V}(\mathbf{b}, \mathbf{r})=\frac{1}{\alpha_{s}\left(\mu_{\text {soft }}^{2}\right)} \int \frac{d^{2} \Delta_{\perp}}{(2 \pi)^{2}} \sqrt{x_{P}} \mathscr{F}_{g}^{\text {off }}\left(x_{P}, \Delta_{\perp}^{2}\right)\left\{e^{-i \mathbf{r} \Delta_{\perp}}-e^{i \mathbf{r} \Delta_{\perp}}\right\} e^{i \mathbf{b} \Delta_{\perp}} .
$$

Here, $\mathscr{F}_{g}^{\mathrm{off}}\left(x_{P}, \Delta_{\perp}^{2}\right)$ is the generalized unintegrated gluon distribution function (UGDF), which can be related with the integrated gluon PDF $x_{P} g\left(x_{P}, \mu_{F}^{2}\right)$ as discussed in Ref. [可], and $\mu_{\text {soft }} \ll \mu_{F}$ is the typical soft scale of the process given by the gluon virtuality $\sim \Delta_{\perp}^{2}$. The QCD coupling at this scale $\alpha_{s}\left(\mu_{\text {soft }}\right)$ is calculated in the framework of the singularity-free Analytic Perturbation Theory and fixed to be $\sim 0.7$ at $\mu_{\text {soft }} \sim \Lambda_{Q C D}$ [目].

At large- $M_{X}$, the gluon emission may be important. In principle, gluons may be radiated from both the $q \bar{q}$ dipole and the hard gluon. The gluons emitted from the quarks are dominantly soft and move collinearly with the quarks, and do not significantly change the invariant mass of the final system $X$. Large $M_{X}$ is instead obtained by gluon emission from the hard gluon, as illustrated in

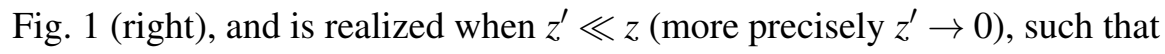

$$
M_{X}^{2} \simeq M_{q \bar{q}}^{2}+\frac{p_{\perp}^{2}}{z^{\prime}} \gg M_{q \bar{q}}^{2}, \quad M_{q \bar{q}}^{2} \simeq \frac{k_{\perp}^{2}+m_{q}^{2}}{z(1-z)} .
$$

Also, due to momentum conservation, the maximal $M_{X}$ at fixed $z^{\prime}$ occurs in the limit $p_{\perp} \sim$ $k_{1, \perp} \gg k_{2, \perp}$, which corresponds to $r_{1} \ll r_{2}$ in impact parameter space. It turns out that the $q \bar{q} g$ and 
$q \bar{q}$ dipole contributions in this limit saturate to the same value, i.e. $M_{q \bar{q} g}^{\text {soft }} \simeq M_{q \bar{q}}^{\text {soft }}$ at large invariant masses $M_{X}[$ [ $]$. In particular, this means that the scattering of the $q \bar{q} g$ system off the proton in the considered asymptotics $M_{X} \rightarrow \infty$ can not be reduced to the scattering of the gluonic dipole.

In order to calculate the $q \bar{q} g$ contribution to the diffractive structure function we include a DGLAP splitting of the hard gluon (with longitudinal momentum fraction $x_{P}$ ) into two gluons one carries momentum fraction $z_{g} x_{P}$ and couples to the hard part, and one is on-shell and contributes to the final state. The diffractive structure function corresponding to the $q \bar{q} g$ contribution can be then written as

$$
x_{P} F_{q \bar{q} g}^{D(4)} \simeq \frac{1}{N_{c}^{2}} \int \frac{d t_{g} d z_{g}}{t_{g}+m_{g}^{2}} \hat{P}_{g g}\left(z_{g}\right) \frac{\alpha_{S}\left(t_{g}\right)}{2 \pi} x_{P} F_{q \bar{q}}^{D(4)}
$$

where the integral is regulated in the infrared by the effective gluon mass $m_{g} \simeq \Lambda_{\mathrm{QCD}}$ in the gluon propagator.

\section{Numerical results}

The HERA data [ [ [ 미] on DDIS are given in the form of the reduced cross section

$$
x_{P} \sigma_{r}^{D(3)}=x_{P} F_{q \bar{q}, T}^{D(3)}+\frac{2-2 y}{2-2 y+y^{2}} x_{P} F_{q \bar{q}, L}^{D(3)}+x_{P} F_{q \bar{q} g}^{D(3)}
$$

expressed in terms of the diffractive structure functions $F_{L, T}^{D(3)}\left(x_{P}, Q^{2}, \beta\right)$. The momentum transfer $t$ is integrated over since in most of the data the leading proton is not observed, and diffraction is equivalently defined through a large rapidity gap. The kinematical variable $y=Q^{2} /\left(s x_{B}\right) \leq$ 1 , where $\sqrt{s}=318 \mathrm{GeV}$ is the center-of-mass energy of ep-collisions in HERA. In Fig. $\square$ we compare the latest ZEUS data [四] with the numerical evaluation of our model. A generally very good agreement is found with CTEQ6L1 gluon PDF [ [ $]$, except for very small values of $Q$ and $M_{X}$, where the QCD factorization scale $\mu_{F}$ becomes very small.

\section{Conclusion}

We have developed a proper QCD framework for diffractive hard scattering, which contains both hard and soft dynamics. The hard part produces a well-defined state of emerging partons, and the soft part is the rescattering of these partons with the color field of the proton remnant.

Numerical evaluation of the analytical results gives good agreement with the precise HERA data on the diffractive deep inelastic cross section. The $q \bar{q}$ contribution is indeed dominant, but at $\beta \lesssim 0.2$, the $q \bar{q} g$ contribution is important. While CTEQ6L1 works well at large $\mu_{F}$ scales and large $x_{P}$, at very small $x_{P} \lesssim 5 \times 10^{-4}$ and scales $\mu_{F}^{2} \sim 1 \mathrm{GeV}^{2}$ the gluon density in the proton is very poorly known and gives a complication in the comparison with the few HERA data points in this extreme region. Standard up-to-date parametrizations have a too low gluon density in this $x_{P}, \mu_{F}^{2}$ region, whereas, e.g., the old GRV94 gluon density does better. Since the diffractive cross section depends directly on the gluon density, and not only indirectly via DGLAP evolution as for inclusive DIS, one here obtains an interesting possibility to constrain the gluon density at very small $x$. 


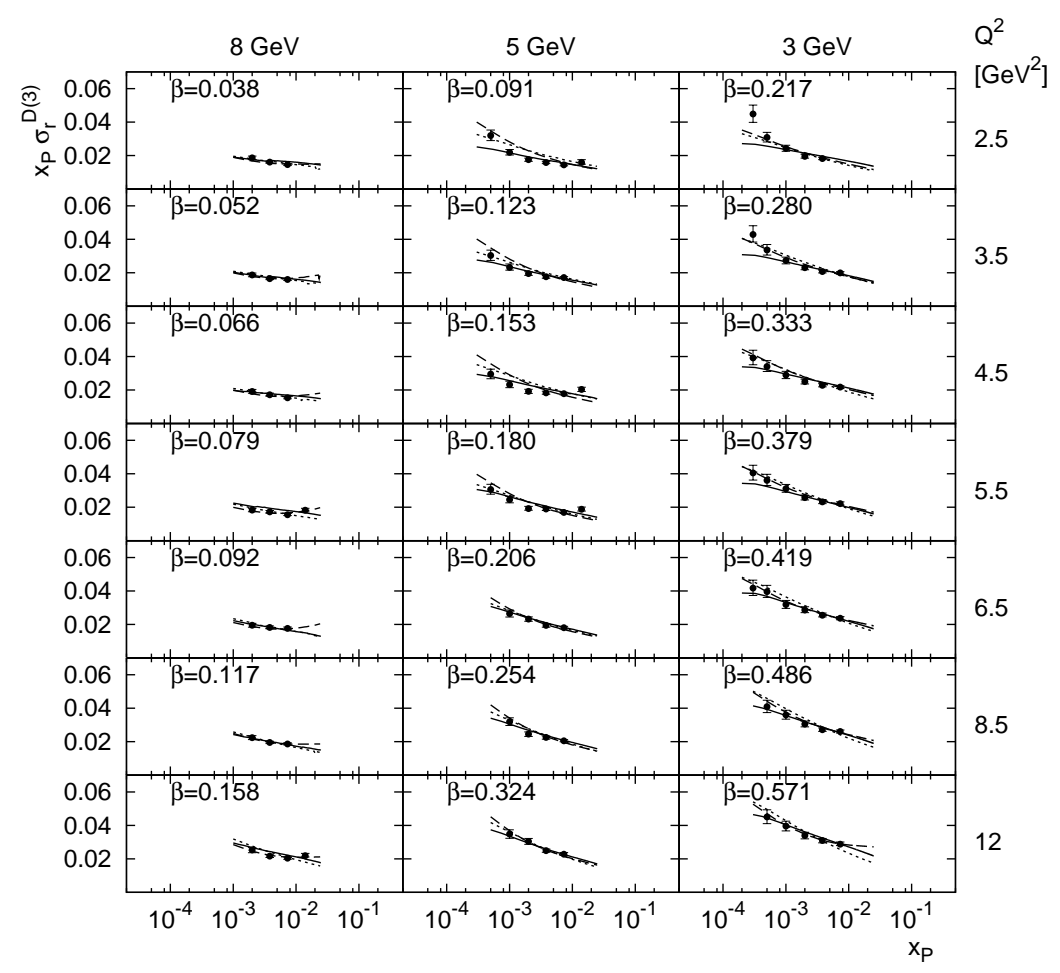

Figure 2: The reduced cross section $x_{P} \sigma_{r}^{D(3)}\left(x_{P}, \beta, Q^{2}\right)$ as a function of $x_{P}$ in the region of low scales $M_{X} \leq 3,5,8 \mathrm{GeV}$ and $Q^{2}<12 \mathrm{GeV}^{2}$, where the lowest $x_{P}$-values are reached. ZEUS data [四] are compared to the model results using different extrapolations of the gluonic density at small $x_{P}$ and $\mu_{F}^{2}$ as detailed in Ref. [焑].

The new model discussed here has a good potential to be applied in many different exclusive and diffractive processes. Having demonstrated that our theoretical formalism for DDIS does describe HERA data, one may then extract the part describing the multigluon exchange process and apply it to other hard processes, including $p \bar{p}$ collisions at Tevatron and LHC.

\section{References}

[1] S. Chekanov et al. [ZEUS Collaboration], Nucl. Phys. B 816, 1 (2009).

[2] M. Derrick et al., Phys. Lett. B 315, 481 (1993); Phys. Lett. B 346, 399 (1995); T. Ahmed et al., Nucl. Phys. B 429, 477 (1994); Nucl. Phys. B 435, 3 (1995).

[3] A. Edin, G. Ingelman and J. Rathsman, Phys. Lett. B 366, 371 (1996); Z. Phys. C 75, 57 (1997).

[4] A. Edin, G. Ingelman and J. Rathsman, arXiv:hep-ph/9912539, in proc. 'Monte Carlo generators for HERA physics', DESY-PROC-1999-02 p. 280; R. Enberg, G. Ingelman and N. Tîmneanu, Phys. Rev. D 64, 114015 (2001); A. Edin, G. Ingelman and J. Rathsman, Phys. Rev. D 56, 7317 (1997); D. Eriksson, G. Ingelman and J. Rathsman, Phys. Rev. D 79, 014011 (2009).

[5] R. Pasechnik, R. Enberg and G. Ingelman, arXiv:1004.2912 [hep-ph].

[6] R. Pasechnik, R. Enberg and G. Ingelman, arXiv:1005.3399 [hep-ph].

[7] J. Pumplin et al., JHEP 0207, 012 (2002). 